\title{
Numerical study of two ill-posed one phase Stefan problems
}

\author{
Julian M. Back ${ }^{1} \quad$ Scott W. McCue ${ }^{2}$ \\ Timothy J. Moroney ${ }^{3}$
}

(Received 28 January 2011; revised 24 June 2011)

\begin{abstract}
We treat two related moving boundary problems. The first is the illposed Stefan problem for melting a superheated solid in one Cartesian coordinate. Mathematically, this is the same problem as that for freezing a supercooled liquid, with applications to crystal growth. By applying a front-fixing technique with finite differences, we reproduce existing numerical results, concentrating on solutions that break down in finite time. This sort of finite time blow-up is characterised by the speed of the moving boundary becoming unbounded in the blowup limit. The second problem, which is an extension of the first, is proposed to simulate aspects of a particular two phase Stefan problem with surface tension. We study this novel moving boundary problem numerically, and provide results that support the hypothesis that it exhibits a similar type of finite time blow-up as the more complicated two phase problem. The results are unusual in the sense that it appears
\end{abstract}

http://anziamj . austms.org.au/ojs/index.php/ANZIAMJ/article/view/3937 gives this article, (C) Austral. Mathematical Soc. 2011. Published July 22, 2011. ISSN 1446-8735. (Print two pages per sheet of paper.) Copies of this article must not be made otherwise available on the internet; instead link directly to this URL for this article. 
the addition of surface tension transforms a well-posed problem into an ill-posed one.

\section{Contents}

1 Introduction

C431

2 Ill-posed superheated Stefan problem

C437

3 One dimensional problem with 'surface tension'

C440

4 Discussion

C443

References

C445

\section{Introduction}

We begin by considering the dimensionless, one phase, Stefan problem for a superheated solid:

$$
\begin{array}{ll}
\text { in } 0<x<s(t), & \frac{\partial v}{\partial t}=\frac{\partial^{2} v}{\partial x^{2}} ; \\
\text { on } x=0, & \frac{\partial v}{\partial x}=0 ; \\
\text { on } x=s(t), & v=0 ; \\
\text { on } x=s(t), & \frac{\partial v}{\partial x}=\beta \frac{d s}{d t} ; \\
\text { at } t=0, & v=V(x)>0, \quad s=1 ;
\end{array}
$$

where $v(x, t)$ is the temperature of the solid phase in $0<x<s(t)$, and $x=s(t)$ is the moving boundary that separates the solid and liquid phases. 
In this model the melting temperature is constant (it is scaled to be $v=0$ ), a condition that is forced via the interface condition (3). The outer liquid phase $x>s(t)$ is ignored as it is assumed the temperature there remains at the melting temperature for all time (using $\mathfrak{u}(x, \mathfrak{t})$ to denote temperature in the liquid phase, this corresponds to $\boldsymbol{u} \equiv 0$ ). The Stefan condition (4) arises from balancing the heat flowing in and out of the interface. Here, the Stefan number $\beta=\mathrm{L} / \mathrm{c}_{\mathrm{v}} \hat{v}$ is a measure of the latent heat absorbed by the molecules during the melting process, where $\mathrm{L}$ is the latent heat per unit mass at the equilibrium temperature, $\boldsymbol{c}_{\mathrm{v}}$ is the specific heat of the solid and $\hat{v}$ is a representative temperature scale.

As the initial temperature $\mathrm{V}(\mathrm{x})$ is greater than the melting temperature $v=0$, the solid is superheated. During the melting process, the heat energy initially contained within the solid causes the interface to retreat and the solid region to shrink [1,2]. This is unlike the classical case in which $V<0$ and the interface advances rather than retreats as the liquid freezes. Superheating arises in a variety of physical processes involving pure materials, for example when melting a block of ice with light, regions can become superheated before any melting occurs. Other examples arise in industrial applications such as electrical welding. Importantly, (1)-(5) is mathematically equivalent to the freezing of a supercooled liquid (simply replace $v$ with $-v$ ), which has applications in crystal growth and has been well studied [3, 4, 5].

The problem (1)-(5) is known to be ill-posed and exhibits finite time blow-up under certain initial conditions. Solutions are categorised into one of the following three cases.

1. Incomplete melting of the solid. The solution for $v$ exists for all time with $s \rightarrow s_{a}^{+}>0$ and $v(x, t) \rightarrow 0^{+}$(for all $0<x<s_{a}$ ) as $t \rightarrow \infty$. Thus, in the limit $t \rightarrow \infty$, there is solid for $0<x<s_{a}$ and liquid for $x>s_{a}$ with both regions at the melting temperature $v=u=0$.

2. Complete melting of the solid. There is a finite time $t_{e}$ such that $s\left(t_{\mathrm{e}}\right)=0$. 
3. Finite time blow-up. The solution exists for $0<t<t_{c}$ and has $s \rightarrow s_{c}^{+}$ and $\dot{s} \rightarrow-\infty$ in the blow-up limit $t \rightarrow t_{c}^{-}$.

An important quantity that characterises qualitative behaviour is the initial superheating parameter

$$
Q=\int_{0}^{1}(V(x)-\beta) d x=\int_{0}^{1} V(x) d x-\beta
$$

The first term on the right-hand side of (6) is the amount of (dimensionless) heat that is required to be removed from the solid in order to reduce the temperature from $v=\mathrm{V}(\mathrm{x})$ to $v=0$. The second term represents the latent heat which must be absorbed by the molecules to melt the solid. Due to the no-flux boundary condition (2), the only energy available to act as latent heat energy (and hence melt the solid) is that initially in the solid.

The relationship between $\mathrm{Q}, \mathrm{V}(\mathrm{x})$ and cases $1-3$ is well known $[1,2,3,6]$. Case 3 will always occur if $Q>0$, as the initial heat energy in the solid is greater than what is required to melt it. It is the surplus energy that leads to blow-up.

If $V(x)$ is smooth with $V(1)=0$ and $V(x)<\beta$ for $0 \leqslant x<1$, then $Q<0$ and case 1 always occurs. Physically, there is not enough heat initially in the solid to convert to the latent heat required to melt the entire solid. As all the heat energy is converted to latent heat energy, the temperature goes to zero and the melting process stops.

A subtly different scenario occurs if $V(x)$ is smooth with $V(1)=0$ and $V(x)$ is monotonic; then $\mathrm{Q}<0$ leads to case 1 and $\mathrm{Q}=0$ leads to case 2. Here the (borderline) case $\mathrm{Q}=0$ corresponds to a situation in which there is precisely the correct amount of initial heat to melt the entire solid and the region completes melting in a finite time.

There are other cases. For example [1, 4], even if $\mathrm{Q}<0$, case 3 may still occur if $\mathrm{V}(x)>\beta$ for some $x \in[0,1]$, or if the heat is initially concentrated near $x=s(0)$. This unusual scenario occurs because heat builds up at the interface and cannot diffuse away fast enough to prevent blow-up. 
The finite time blow-up exhibited when $\mathrm{Q}>0$ is what we are most interested in. The form of the blow-up, derived by both Herrero and Velázquez [5] and King and Evans [7], is

$$
s(t)-s_{c} \sim 2\left(t_{c}-t\right)^{1 / 2} \ln ^{1 / 2}\left[-\ln \left(t_{c}-t\right)\right] \quad \text { as } t \rightarrow t_{c}^{-} .
$$

This article solves (1)-(5) numerically using front-fixing and the method of lines [8]. Other common computational approaches include a conservative finite difference scheme [9], a Petrov-Galerkin finite element approach [10] or the enthalpy method [8, and references], the latter is only applicable when constant boundary data is prescribed. The results are presented in Section 2, where we investigate the effects of varying $Q$ on the numerical solution and confirm that for $\mathrm{Q}>0$ the numerical solution ceases to exist at a finite time.

The second problem considered in this article relates to the two phase Stefan problem for a melting sphere with surface tension:

$$
\begin{array}{ll}
\text { in } s(t)<r<1, & \frac{\partial u}{\partial t}=\frac{\partial^{2} u}{\partial r^{2}}+\frac{2}{r} \frac{\partial u}{\partial r} ; \\
\text { in } 0<r<s(t), & \frac{\partial v}{\partial t}=\frac{\partial^{2} v}{\partial r^{2}}+\frac{2}{r} \frac{\partial v}{\partial r} ; \\
\text { on } r=0, & \frac{\partial v}{\partial r}=0 ; \\
\text { on } r=s(t), & u=v=\sigma\left(1-\frac{1}{s}\right) ; \\
\text { on } r=s(t), & \frac{\partial u}{\partial r}-\frac{\partial v}{\partial r}=-\beta \frac{d s}{d t} ; \\
\text { on } r=1, & u=1 ; \\
\text { at } t=0, & v=V, \quad s=1 ;
\end{array}
$$

where $u$ and $v$ are the temperature in the liquid and solid regions, respectively, and $\beta$ is again a Stefan number. The interface condition (11), which is often referred to as the Gibbs-Thomson condition, states that the melting temperature of the solid ball is not constant, but instead decreases with 
particle size. Here the dimensionless surface tension parameter $\sigma$ acts on the curvature of the interface, which for a sphere is $1 / \mathrm{s}$. Typically $\sigma$ is sufficiently small that the Gibbs-Thomson effect is relevant only for micro and nanosized particles. For the case $\sigma=0,(8)-(14)$ reduces to the classical two phase Stefan problem for a melting sphere, and is known to be well-posed [11].

The two phase problem (8)-(14) is treated in [12, 13]. Numerical solutions show that as melting proceeds, the interface $r=s(t)$ moves towards the centre of the ball, and the melting temperature decreases according to (11). For a given set of parameter values, there is a point in time after which the solid is locally superheated, with the temperature everywhere in the solid greater than the melting temperature (although less than the bulk melting temperature for a flat interface). This behaviour is illustrated in Figure 1 [12]. In Figure 1(a), the fifth profile (the one furthest to the left) shows this apparent self-superheating in the solid phase. For subsequent times, as seen in Figure 1(b), the flux of heat at the solid-melt interface increases very quickly, and the solution appears to have a form of finite time blow-up at $t=t_{c}$ which is accompanied by the interface speed $d s / d t \rightarrow-\infty$ as $t \rightarrow t_{c}^{-}$. We emphasise that the apparent existence of finite time blow-up for (8)-(14) is unusual because surface tension is often associated with regularising singular behaviour. In this case the problem without surface tension $(\sigma=0)$ is known to be well-posed, and it is the addition of surface tension that appears to lead to blow-up.

Figure 1(b) shows the temperature in the outer liquid phase hugging the melting temperature curve near blow-up. McCue et al. [12] use this observation to suggest that $u \sim \sigma(1-1 / r)$ for $r-s(t) \ll 1$ as $t \rightarrow t_{c}^{-}$, which leads to a novel one phase problem that they leave for further research. In Section 3 of the present study, we consider the one dimensional ('toy') version of this novel moving boundary problem, and note that when the surface tension parameter vanishes, the problem reduces to the ill-posed superheated Stefan problem (1)-(5). Indeed, McCue et al. [12] observe that blow-up for (8)-(14) may be of the same form as that for (1)-(5). Section 3's brief numerical study of the toy problem supports this view, and suggests the matter is worth 

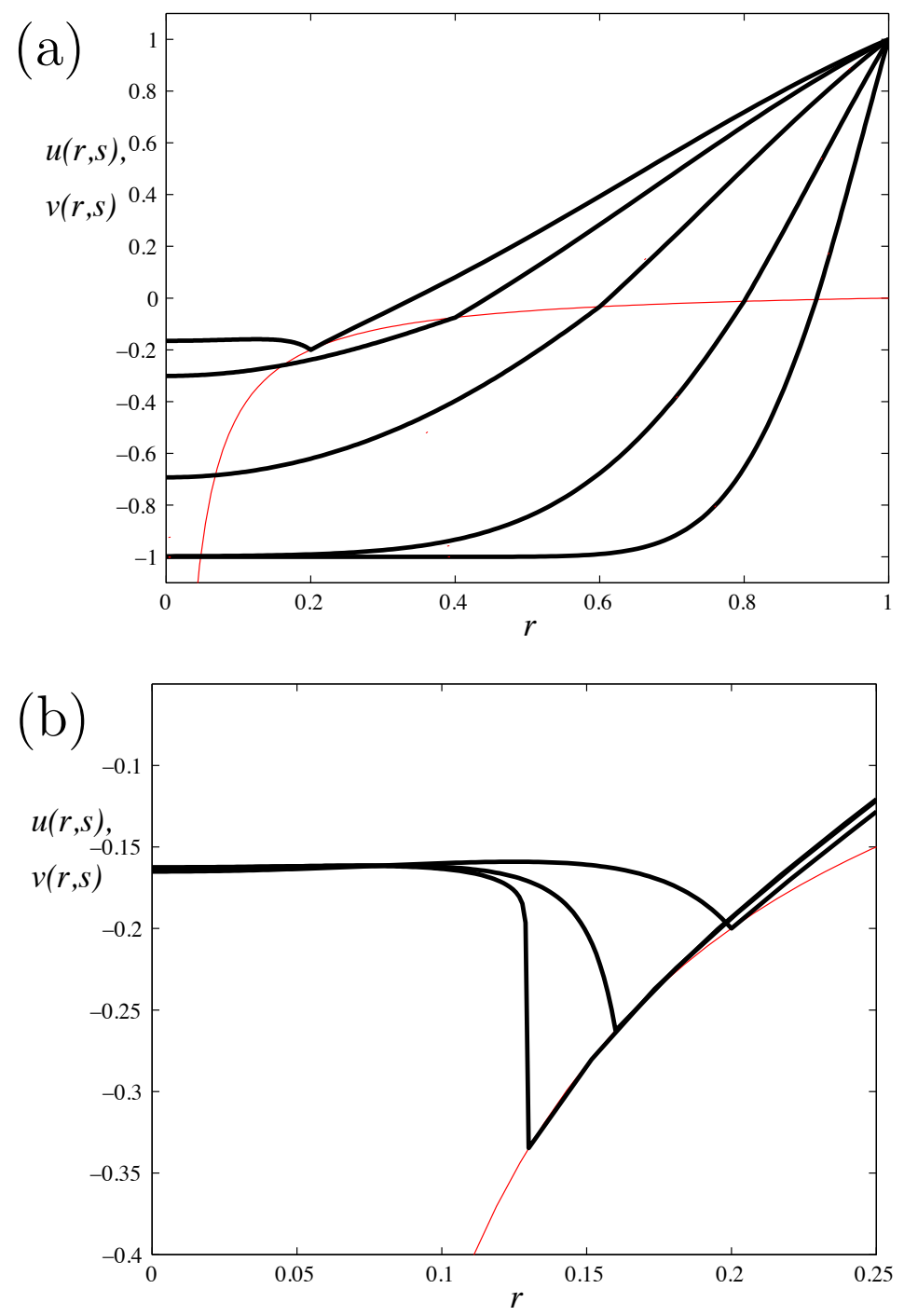

Figure 1: Numerical solutions to (8)-(14) with $\beta=0.1, \mathrm{~V}=-1$ and $\sigma=0.05$, as calculated by McCue et al. [12]. The thick curves represent temperature profiles: (a) from right to left the profiles are for $t=0.0088$, $0.0292,0.0769,0.0114$ and 0.1306 ; (b) the associated times from right to left are $0.1306,0.1312$ and 0.1314 . The thin red curve denotes the melting temperature (11). Reproduced [12] by permission of Oxford University Press. 
further consideration.

\section{Ill-posed superheated Stefan problem}

This section solves the superheated Stefan problem (1)-(5) computationally by applying the method of lines. The numerical results are used to investigate the effects of varying $Q$ and to demonstrate the behaviour of cases 1-3.

We first introduce the transformation $\xi=x / s(t)$ due to Landau [14]. This has the effect of fixing the domain of $(1)-(5)$ to $0 \leqslant \xi \leqslant 1$. A standard finite difference scheme on a uniformly spaced mesh is used to discretise the transformed equations in space.

Second order central differences discretise the second derivative corresponding to the diffusion term in the transformed equation (1). A consequence of the Landau transformation is the introduction of an advection-like term in this equation. We also use second order central differences to discretise this term, recognising that this restricts the scheme to operating on fine spatial meshes in order to ensure the solution is free of numerical oscillations. The transformed equations (2) and (3) for the boundary conditions are treated in standard fashion. The spatial derivative in the transformed equation (4) is discretised using a second order backward difference, since it is evaluated at the right boundary $\xi=1$.

The transformed and discretised forms of (1) (subject to (2) and (3)) and (4) form a semidiscrete system of ordinary differential equations (ODEs) for the temperature at each spatial node and the position of the moving boundary. We solve this system of ODEs, subject to the initial condition (5), using the MATLAB solver ode15s. This method-of-lines approach frees us from being directly concerned with stability requirements, as the solver itself adaptively chooses both the time-step size and the order of the temporal scheme to ensure that local error tolerances are met for each time-step [15]. The efficiency of 


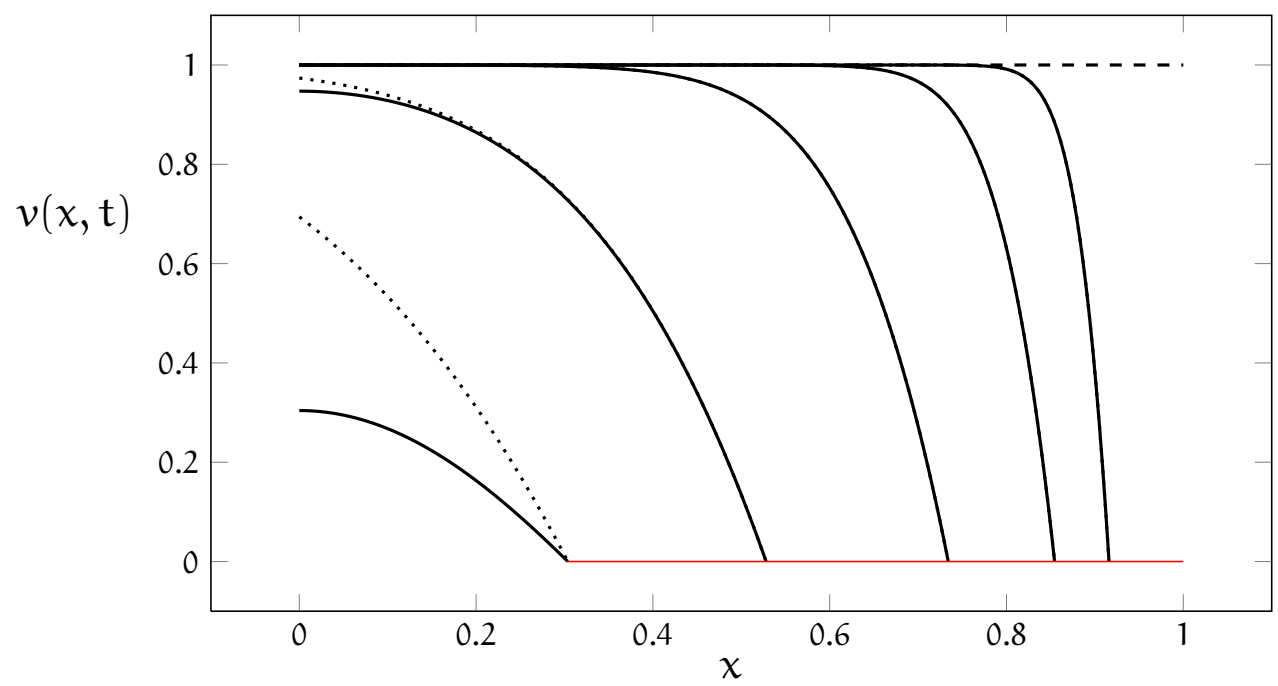

Figure 2: Temperature profiles for (1)-(5) with $V=1$ and $\beta=1.35$. The numerical solutions and the small time approximation are indicated by thick solid and dotted curves, respectively, and are calculated at $t=0.002,0.006$, $0.020,0.063$ and 0.150 (right to left). The thin horizontal red line is the temperature in the liquid region, which is everywhere zero for this problem.

the implicit scheme employed by ode15s is improved by passing it the known sparsity pattern of the Jacobian matrix.

The problem was solved on a sequence of refined meshes, until convergence to a grid independent solution was obtained. We found that 20,000 mesh nodes sufficed, requiring a run time of approximately 15 seconds on a standard desktop machine.

As a test for accuracy, the numerical solutions are compared with a small time solution. Problem (1)-(5) does not have an exact solution, but if we assume the initial temperature $\mathrm{V}(\mathrm{x}) \equiv$ constant, and replace (2) with the condition $v \rightarrow \mathrm{V}$ as $\mathrm{x} \rightarrow-\infty$, then there is an well-known exact solution called the Neumann solution [16]. This exact solution provides the small time 
behaviour for (1)-(5) when $\mathrm{V}(\mathrm{x}) \equiv$ constant, and may be used to check our calculations. Our comparison is shown in Figure 2 which, for small times, shows the Neumann solution approximates the numerical solution very well, suggesting that the numerical solutions are accurate in that regime. As expected, the comparison is not valid for larger times.

We use the initial condition

$$
V(x)= \begin{cases}a, & 0<x<\alpha, \\ a-a \frac{(x-\alpha)(x-\gamma)}{(1-\alpha)(1-\gamma)}, & \alpha<x \leqslant 1,\end{cases}
$$

which for $\alpha=0.2, \gamma=0.9$ and $a=0.4,0.5,0.6$ and 0.7 corresponds to $\mathrm{Q}=-0.33,-0.167,-1.3 \times 10^{-7}$ and 0.33 , respectively. This condition with these values was used by King and Evans [7], where numerical solutions to (1)-(5) are also provided.

Figure 3 plots $\mathbf{s}(\mathbf{t})$ for the same parameter values as in [7, Figure 5(a)]. The values $Q=-0.33$ and -0.167 correspond to case 1 described in Section 1, for which the interface $s \rightarrow s_{a}^{+}$as $t \rightarrow \infty$. The next curve for $Q=-1.3 \times 10^{-7}$ is extremely close to the borderline case 2, but exhibits the same qualitative behaviour as the previous two curves. Finally, the solution for $\mathrm{Q}=0.33$ results in case 3 , and has finite time blow up with $\mathrm{s} \rightarrow \mathrm{s}_{\mathrm{c}}^{+}, \dot{\mathrm{s}} \rightarrow-\infty$ as $t \rightarrow t_{c}^{-}$. Our scheme predicts the critical time to be roughly $t_{c}=0.0072$; however, the problem is ill-posed, so these calculations are understandably delicate (indeed, the scheme of King and Evans [7] does not appear to capture the blow-up as well as ours).

Figure 4 shows a temperature profile very close to blow up. Beyond the critical time numerical errors begin to appear and the solution exhibits unreasonable behaviour such as spikes and jumps. Figure 4(b) compares the asymptotic prediction derived by King and Evans [7]. Given the ill-posedness of the problem, the agreement is very good this close to blow-up. 


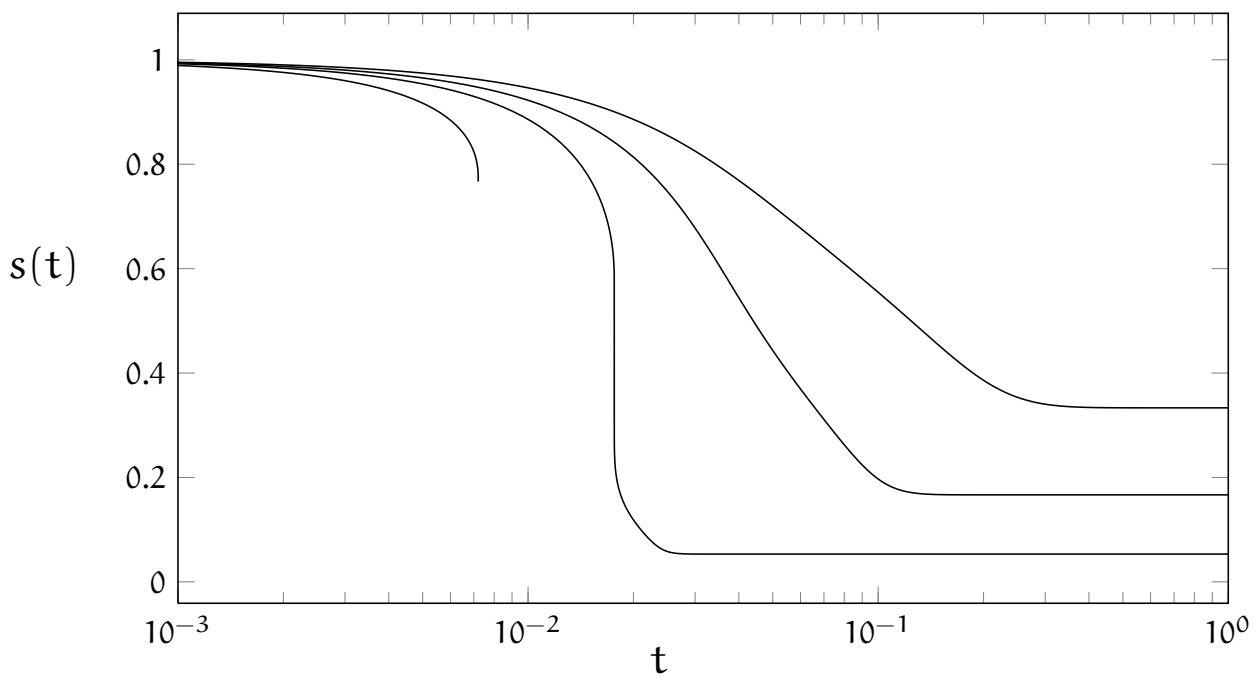

FiguRE 3: The interface position $s(t)$ for $\beta=1$ with the initial condition (15). From top to bottom, $\mathrm{Q}=-0.33,-0.167,-1.3 \times 10^{-7}$ and 0.33 . The top two solutions, with $\mathrm{Q}=-0.33$ and -0.167 , approach a constant value, as described in case 1. The fourth solution exhibits finite time blow-up, as described in case 3 , occurring at approximately $t_{c}=0.0072$.

\section{One dimensional problem with 'surface tension'}

In this section we consider the novel one phase Stefan problem:

$$
\begin{array}{ll}
\text { for } 0<x<s(t), & \frac{\partial^{2} v}{\partial x^{2}}=\frac{\partial v}{\partial t} ; \\
\text { on } x=0, & \frac{\partial v}{\partial x}=0 ; \\
\text { on } x=s(t), & \frac{\partial v}{\partial x}=\beta \frac{d s}{d t}+\frac{\sigma}{s^{2}} ;
\end{array}
$$




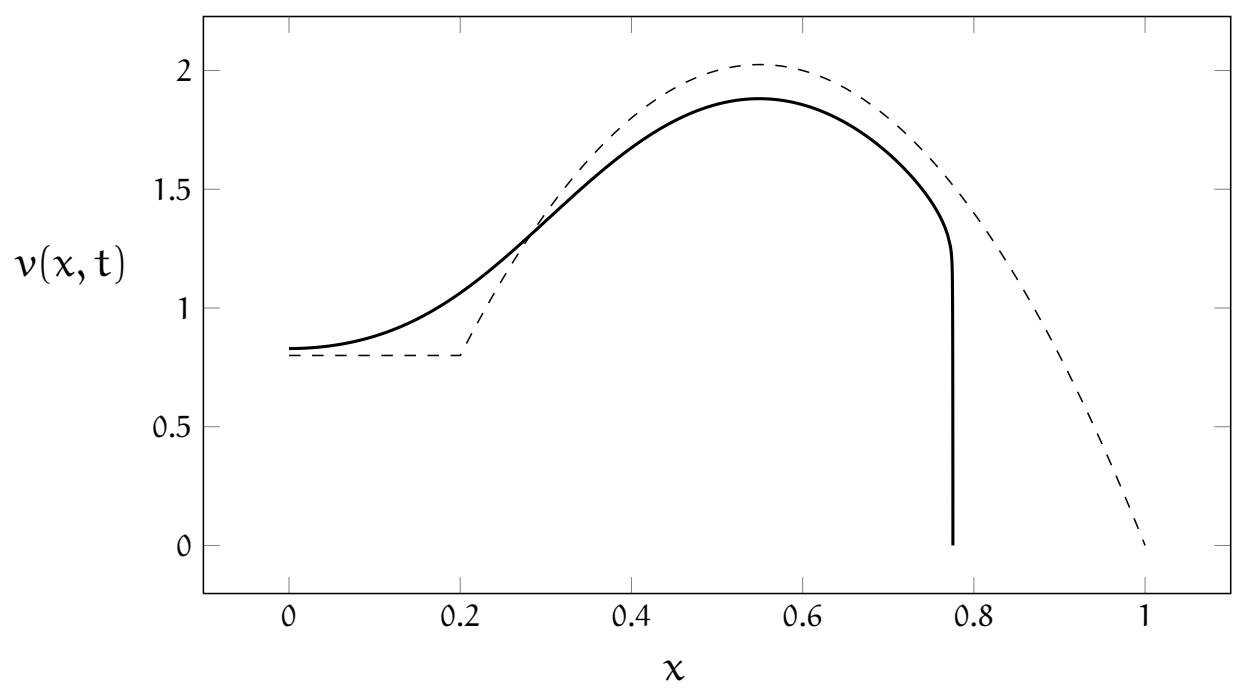

(a)

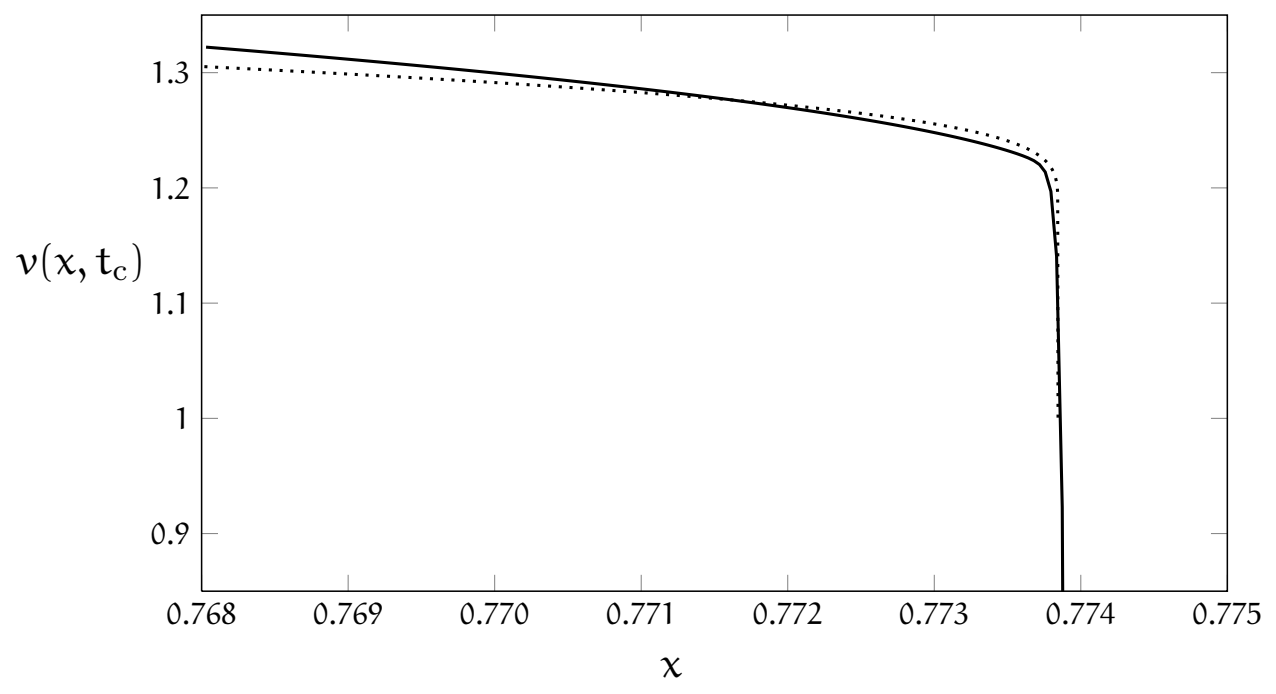

(b)

Figure 4: (a) The numerically calculated near-blow-up profile (solid) for $\beta=$ $1, t_{c}=0.0072$. The initial condition (dashed) (15) with $Q=-0.33$. (b) A close-up of the near-blow-up profile (solid) compared to the asymptotically predicted blow-up profile (dotted) derived by King and Evans [7]. 


$$
\begin{array}{ll}
\text { on } x=s(t), & v=\sigma\left(1-\frac{1}{s}\right) ; \\
\text { at } \mathrm{t}=0 \text { for } 0 \leqslant x \leqslant 1, & s=1 ; \quad v=V(x) \geqslant 0 ; \\
\text { in } s(t)<x<1, & u(x, t)=\sigma\left(1-\frac{1}{x}\right) .
\end{array}
$$

As mentioned in Section 1, this is the one dimensional version of a problem suggested by McCue et al. [12, Discussion]. The two parameters are $\sigma$, a surface tension parameter, and $\beta$, the Stefan number. The case $\sigma=0$ reduces (16)-(21) to (1)-(5).

Of course surface tension does not act on one dimensional interfaces, and the actual problem suggested by McCue et al. [12] has (16) replaced with the radially symmetric version of the heat equation, and independent variable $x$ in (16)-(21) replaced with $\boldsymbol{r}$. But the toy problem (16)-(21) offers a great deal of insight into the eventual behaviour of solutions of (8)-(11). Equations (16)(20) are solved with the same numerical scheme described in Section 2, with minor adjustments.

In Figure 5, the evolution of the interface $x=s(t)$ is shown for $\beta=1$ and the initial condition (15) with $\mathrm{Q}=-0.33$. The curve for $\sigma=0$ is the same as the top curve in Figure 3. Here the solution exists for all time with $s \rightarrow s_{a}^{+}$ as $t \rightarrow \infty$, as described by case 1 in Section 1 . However, when we introduce surface tension $\sigma>0$, the qualitative behaviour changes, and the solution exhibits finite time blow-up in a manner that is consistent with case 3 , with the critical time $t_{c}$ decreasing as $\sigma$ increases. Our conclusion is that (16)-(21) appears to be ill-posed for all $\sigma>0$, with the solutions exhibiting finite time blow-up regardless of the initial conditions.

Finally, Figure 6 shows temperature profiles near blow-up calculated for $\beta=1$ and $\sigma=0.05$. These appear qualitatively similar to those in Figure 1, supporting the idea that the toy problem (16)-(21) can be used to model behaviour of the more complicated two phase problem (8)-(14). The initial condition used for this figure is $\mathrm{V}(\mathrm{x}) \equiv 0$, which provides a strong indication 


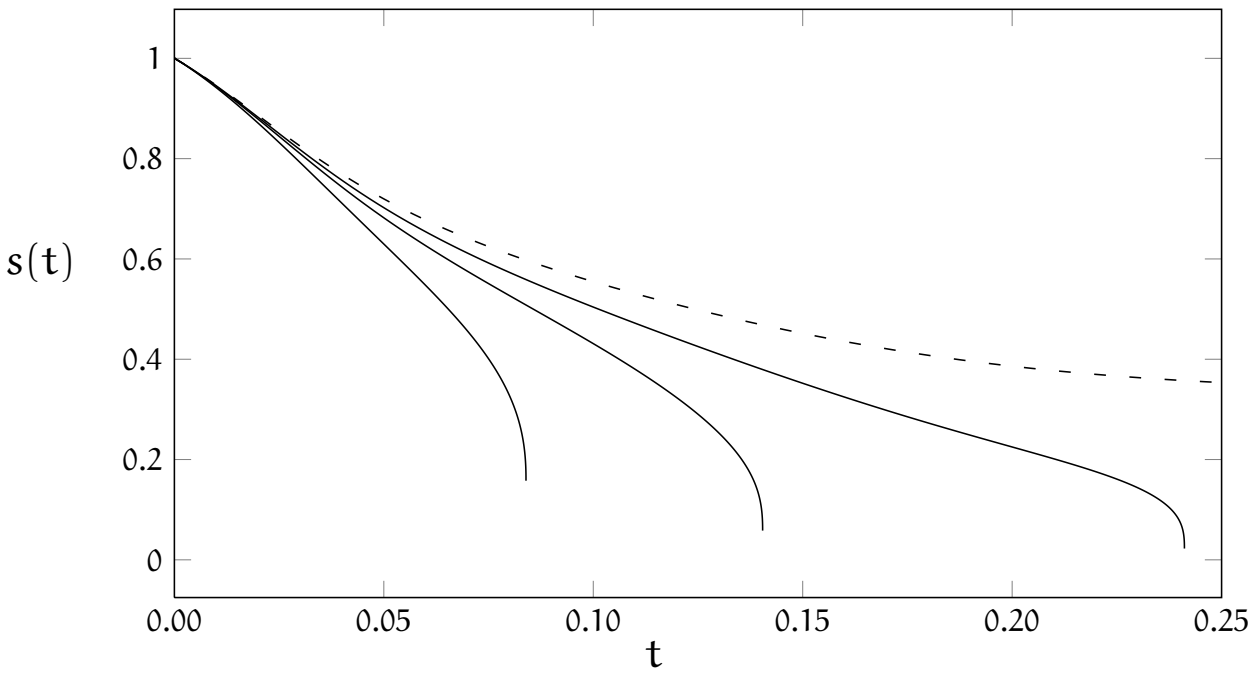

FiguRE 5: The interface position calculated for $\beta=1$ and the initial condition (15) with $\mathrm{Q}=-0.33$. From top to bottom, the curves are for $\sigma=0$ (dashed), 0.05, 0.1 and 0.2 .

that blow-up is inevitable for (16)-(21), since the larger $\mathrm{V}(\mathrm{x})$ is, the more likely blow-up is to occur.

\section{Discussion}

We revisited the ill-posed superheated Stefan problem (1)-(5) and produced numerical solutions that are accurate and consistent with previous results and observations. Subsequently, we adapted this numerical scheme to solve (16)(21), which is a novel moving boundary problem with a surface tension-type parameter $\sigma$ and an unusual Stefan condition. For the case $\sigma=0$, the problem (16)-(21) reduces to (1)-(5), and has solutions that blow up in finite time, depending on the Stefan number $\beta$ and the initial condition $v(x, 0)=V(x)$. On the other hand, for $\sigma>0$ our preliminary results suggest 


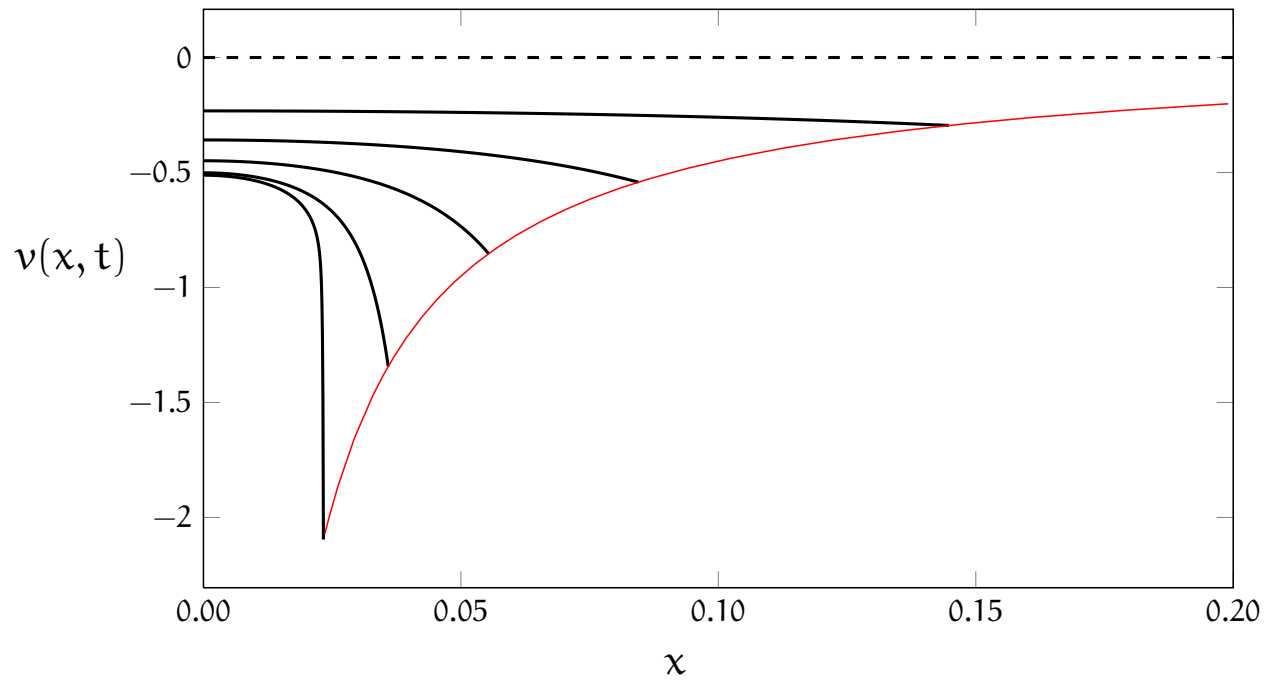

Figure 6: Temperature profiles for $\beta=1$ and $\sigma=0.05$. From right to left the times are $t=6.1886,6.1990,6.2004$ and 6.2007. The dashed line is the initial condition $\mathrm{V}=0$ and the thin red line is the melting temperature (19).

that (16)-(21) is ill-posed with all solutions exhibiting finite time blow-up regardless of $\beta$ and $V(x)$.

Our argument is that the toy problem (16)-(21) illustrates near blow-up behaviour for the two phase Stefan problem (8)-(14) for melting a sphere. The above results suggest that solutions to the more complicated problem (8)(14) also exhibit finite time blow-up regardless of $\beta$ and $V(x)$, provided the surface tension parameter $\sigma>0$. These ideas are worth pursing in the future.

Acknowledgements SWM thanks Prof. John King for fruitful discussions on the two phase Stefan problem (8)-(14). 


\section{References}

[1] S. D. Howison, J. R. Ockendon, and A. A. Lacey. Singularity development in moving-boundary problems. Q. J. Mech. Appl. Math., 38(3):343-360, 1985. doi:10.1093/qjmam/38.3.343 C432, C433

[2] A. A. Lacey and J. R. Ockendon. Ill-posed free boundary problems. Control and Cybernetics, 14:275-296, 1985. C432, C433

[3] A. Fasano and M. Primicerio. General free-boundary problems for the heat equation I. J. Math. Anal. Appl, 57:694-723, 1977. C432, C433

[4] A. Fasano, M. Primicerio, and A. A. Lacey. New results on some classical parabolic free-boundary problems. Quart. Appl. Math., 38:439-460, 1981. C432, C433

[5] M. A. Herrero and J. J. L. Velázquez. Singularity formation in the one-dimensional supercooled Stefan problem. Euro. J. Appl. Math., 7:119-150, 1996. doi:10.1137/04060528X C432, C434

[6] B. Sherman. A general one-phase Stefan problem. Quart. Appl. Math., 28:377-382, 1970. C433

[7] J. R. King and J. D. Evans. Regularization by kinetic undercooling of blow-up in the ill-posed Stefan problem. SIAM J. Appl. Math., 65(5):1677-1707, 2005. doi:10.1137/04060528X C434, C439, C441

[8] J. Crank. Free and moving boundary problems. Clarendon Press, Oxford, 1984. C434

[9] T. C. Illingworth and I. O. Golosnoy. Numerical solutions of diffusion-controlled moving boundary problems which conserve solute. J. Comp. Phys., 209:207-225, 2005. doi:10.1016/j.jcp.2005.02.031 C434

[10] F. Liu and D. L. S. McElwain. A computationally efficient solution technique for moving-boundary problems in finite media. IMA J. Appl. Math, 59:71-84, 1997. doi:10.1093/imamat/59.1.71 C434 
[11] S. W. McCue, B. Wu, and J. M. Hill. Classical two-phase Stefan problem for spheres. Proc. R. Soc. A, 464:2055-2076, 2008. doi:10.1098/rspa.2007.0315 C435

[12] S. W. McCue, B. Wu, and J. M. Hill. Micro/nanoparticle melting with spherical symmetry and surface tension. IMA J. Appl. Math., 74:439-457, 2009. doi:10.1093/imamat/hxn038 C435, C436, C442

[13] B. Wu, S. W. McCue, P. Tillman, and J. M. Hill. Single phase limit for melting nanoparticles. Appl. Math. Model., 33:2349-2367, 2009. doi:10.1016/j.apm.2008.07.009 C435

[14] H. G. Landau. Heat conduction in a melting solid. Quart. J. Appl. Math., 8:81-94, 1950. C437

[15] L. F. Shampine and M. W. Reichelt. The MATLAB ODE Suite. SIAM Journal on Scientific Computing, 18:1-22, 1997. C437

[16] S. C. Gupta. The classical Stefan problem: Basic concepts, modelling and analysis. Elsevier, Amsterdam, 2003. C438

\section{Author addresses}

1. Julian M. Back, Mathematical Sciences, Queensland University of Technology, Brisbane, Queensland 4001, Australia. mailto: j. back@student.qut. edu . au

2. Scott W. McCue, Mathematical Sciences, Queensland University of Technology, Brisbane, Queensland 4001, Australia. mailto:scott.mccue@qut.edu.au

3. Timothy J. Moroney, Mathematical Sciences, Queensland University of Technology, Brisbane, Queensland 4001, Australia. mailto:t.moroney@qut. edu. au 